Original Article

\title{
SIMULTANEOUS METHOD FOR THE ESTIMATION OF BEDAQUILINE AND DELAMANID IN HUMAN PLASMA USING HIGH-PERFORMANCE LIQUID CHROMATOGRAPHY
}

\author{
A. K. HEMANTH KUMAR, V. SUDHA, A. VIJAYAKUMAR, C. PADMAPRIYADARSINI \\ Department of Clinical Pharmacology, National Institute for Research in Tuberculosis, Chennai 600031, India \\ Email: akhemanth20@gmail.com
}

Received: 22 Jan 2021, Revised and Accepted: 19 Apr 2021

\section{ABSTRACT}

Objective: A specific, simple and sensitive high-performance liquid chromatographic method for the estimation of Bedaquiline (BDQ) and Delamanid (DLM) in human plasma was developed.

Methods: The method involved deproteinization and further extracted the analyte using Solid Phase Extraction (SPE) cartridge and analysed using C18 column with the wavelength set at $231 \mathrm{~nm}$. The isocratic mobile phase consisted of $10 \mathrm{mmol}$ ammonium acetate buffer containing $0.25 \%$ acetic acid and $0.02 \%$ trifluoroacetic acid and acetonitrile in the ratio of 20:80(v/v). The validation parameters were evaluated. The method was applied to estimate plasma BDQ and DLM collected from five MDR-TB patients.

Results: Well resolved peaks of BDQ and DLM at retention times of 5.4 and 2.6 min were obtained respectively. The calibration curve was linear over a range of $0.01-10.0 \mu \mathrm{g} / \mathrm{ml}$ for both BDQ and DLM. The intra-and inter-day relative standard deviations for standards were below $10 \%$. The recoveries for BDQ ranged from $101 \%$ to $107 \%$ and $98 \%$ to $107 \%$ for DLM respectively.

Conclusion: A specific and sensitive method for simultaneous determination of BDQ and DLM in plasma using high-performance liquid chromatography was developed. This method can be used in clinical studies to evaluate drug exposure.

Keywords: Bedaquiline, Delamanid, Plasma, HPLC

(C) 2021 The Authors. Published by Innovare Academic Sciences Pvt Ltd. This is an open access article under the CC BY license (https://creativecommons.org/licenses/by/4.0/)

DOI: https://dx.doi.org/10.22159/ijpps.2021v13i6.40853. Journal homepage: https://innovareacademics.in/journals/index.php/ijpps.

\section{INTRODUCTION}

The emergence of drug resistance is a major threat to global tuberculosis (TB) care and control. The crisis of MDR-TB detection and treatment continues. Recognizing that the treatment of pre-XDR and XDR-TB cases are very complex and given the urgent need to increase treatment success to DR-TB, careful evaluation of newer drugs and treatment strategies are urgently needed to build a better, safer, less toxic, shorter and cheaper regimen to reduce patient suffering and mortality. BDQ and DLM are newly approved anti-TB drugs and all are well tolerated. BDQ, previously known as TMC 207 , belongs to a new class of drugs called diarylquinolines and is placed with anti-TB drugs belonging to Group 5, primarily because it does not belong to any other TB drug families. DLM is a pro-drug that must be reduced by the deazaflavin-dependent nitroreductase to its des-nitro metabolite to be active [1]. The World Health Organization (WHO) recommends using these new anti-TB drugs for a standardized duration of $24 \mathrm{w}$ in patients with drug-resistant tuberculosis. The concomitant use of BDQ and DLM is restricted to patients with "no other therapeutic options". Médecins Sans Frontières has supported the use of combination BDQ and DLM for people with few treatment options since 2016. Based on WHO recommendations, Phase III clinical trials are initiated with BDQ and DLM in pre-XDR and XDR pulmonary TB patients.

Several analytical methods have been developed for the estimation of BDQ in plasma which includes high-performance liquid chromatography (HPLC) inductively coupled plasma (ICP), mass spectrometry (MS) [2, 3], tandem mass spectrometry $[4,5]$ and LCMS/MS [6]. Most of the existing methods for BDQ, however, were developed using LCMS/MS. Furthermore, LCMS/MS instrument is quite expensive and may not be possible in resource-limited settings. Similarly, LCMS/MS methods for the determination of DLM in plasma have been developed [7]. There is no simultaneous method for the estimation of BDQ and DLM in plasma using HPLC is available. Therefore, the purpose of this study was to develop a simple, sensitive and specific HPLC method for simultaneous estimation of plasma BDQ and DLM concentrations in MDR-TB patients.

\section{MATERIALS AND METHODS}

Pure BDQ powder was obtained from Janssen Pharmaceuticals (Belgium) and DLM from Otsuka Pharmaceuticals (Japan). Acetonitrile (Merck), orthophosphoric acid, acetic acid, dimethy sulphoxide (DMSO) and trifluoroacetic acid were purchased from Qualigens, India. Deionized water was processed through a water purification system (Siemens, Germany). Pooled human plasma was obtained from a Blood Bank, Chennai, India. All chemicals used in the method were of analytical grade.

A stock BDQ standard solution $(1 \mathrm{mg} / \mathrm{ml}$ ) was prepared by dissolving BDQ in acetonitrile. DLM stock solution was prepared separately by dissolving in DMSO. Working standard concentrations ranging from 0.01 to $10 \mu \mathrm{g} / \mathrm{ml}$ for DLM and BDQ together were prepared in pooled plasma. Calibration standards for BDQ and DLM were freshly prepared on the first day of the validation and stored at-20 ${ }^{\circ} \mathrm{C}$ to carry out the validation parameter experiments on the other validation days.

Samples were prepared by adding $300 \mu$ of $4 \%$ orthophosphoric acid in water to $300 \mu \mathrm{l}$ of calibration standards and test samples in plasma. The microcentrifuge vials were vortexed for $3 \mathrm{~min}$ and centrifuged for $10 \mathrm{~min}$ at 10,000 RPM. The analyte was extracted through solid-phase extraction cartridges (MCX 1 cc $30 \mathrm{mg}$ ). The eluted solution was evaporated to dryness. The dried residue was reconstituted in $100 \mu \mathrm{l}$ of mobile phase and $30 \mu \mathrm{l}$ was injected into the HPLC system.

\section{Chromatographic system}

The HPLC system-Prominence-i (Shimadzu Corporation, Kyoto, Japan) comprised of two pumps (LC-2030-3D), photodiode array detector (SPD-20AV) with a built-in autosampler and system controller. Data collection and acquisition were carried out using Lab solution software. Ascentis R Express C18 (15 cm X 4.6 mm ID, $2.7 \mu \mathrm{gm}$ particle size (Merck, Germany) was used as the analytical column.

An isocratic mobile phase was prepared by mixing $10 \mathrm{mmol}$ ammonium acetate buffer containing $0.25 \%$ acetic acid and $0.02 \%$ 
trifluoroacetic acid and acetonitrile in the ratio of 20:80 (v/v). The solvents were degassed individually using a Millipore vacuum pump before preparing the mobile phase. Each chromatogram was run for $7 \mathrm{~min}$ at a flow rate of $1.0 \mathrm{ml} / \mathrm{min}$ at $40{ }^{\circ} \mathrm{C}$, with the PDA detector set at a wavelength of $231 \mathrm{~nm}$. Unknown concentrations were calculated from linear regression analysis vs BDQ or DLM concentrations curve; linearity was verified using estimates of correlation coefficient ( $\mathrm{r}$ ).

\section{Analytical method validation}

The parameters for validation of the method developed included linearity, inter-and intra-day variations, accuracy and precision, specificity and recovery. The calibration curve for BDQ and DLM consists of 7 standards ranging from $0.01 \mu \mathrm{g} / \mathrm{ml}$ to $10.0 \mu \mathrm{g} / \mathrm{ml}$. The intra-day and inter-day variations were evaluated by processing each sample containing BDQ and DLM standard concentration in duplicate for six consecutive days. The accuracy and linearity were determined by analysing a set of standard solutions with concentrations ranging from 0.01 to $10.0 \mu \mathrm{g} / \mathrm{ml}$. In order to evaluate the precision of the method, plasma samples containing varying concentrations of BDQ and DLM were analyzed in duplicate on three consecutive days.

For the recovery experiments, known concentrations of BDQ and $\operatorname{DLM}(0.05,0.5,1.0$ and $5.0 \mu \mathrm{g} / \mathrm{ml})$ were prepared in pooled human plasma and were spiked with lower and higher concentrations of standards. The percentage of drug recovery from plasma samples was calculated by dividing the difference in BDQ and DLM concentrations by the added concentration. Recovery experiments were carried out on three different occasions.
The effect of endogenous substances was examined by analyzing blank plasma samples using a different batch of pooled plasma. Interference from certain anti-tuberculosis drugs such as rifampicin, isoniazid, pyrazinamide, ethambutol, streptomycin, ethionamide, levofloxacin and certain antiretroviral drugs, namely, nevirapine, efavirenz, zidovudine, didanosine, stavudine, lamivudine, saquinavir, lopinavir, ritonavir and indinavir at a concentration of $10 \mu \mathrm{g} / \mathrm{ml}$ was also evaluated.

Limits of quantitation (LOQ) and detection (LOD) values were estimated mathematically from the standard curve equations. The LOQ was obtained by multiplying the standard deviation (SD) of the Y-axis intercepts by 10 . The LOD was equal to 3.3 times the SD of the Y-axis intercepts [8].

\section{RESULTS}

Under the chromatographic conditions described above, BDQ and DLM were well separated and seen as a discrete peak in the representative chromatograms of extracted plasma standards 10.0 and $0.01 \mu \mathrm{g} / \mathrm{ml}$ and an extracted blank plasma sample from MDR-TB patient (fig. 1A$\mathrm{B})$ with the retention times of 5.4 and $2.6 \mathrm{~min}$. Blank plasma sample did not give any peak at the retention times of BDQ and DLM (fig. 1C). In view of the potent antimycobacterial activity, BDQ and DLM are used in the treatment of MDR-TB along with other second-line antituberculosis drugs. It, therefore, becomes necessary to rule out the interference of other second-line anti-TB drugs as well as anticipated co-administered drugs in the assay of BDQ and DLM and establish the specificity of the method. No endogenous substances, first-line anti-TB drugs, second-line anti-TB drugs or anti-retroviral drugs interfered with the BDQ and DLM chromatogram.

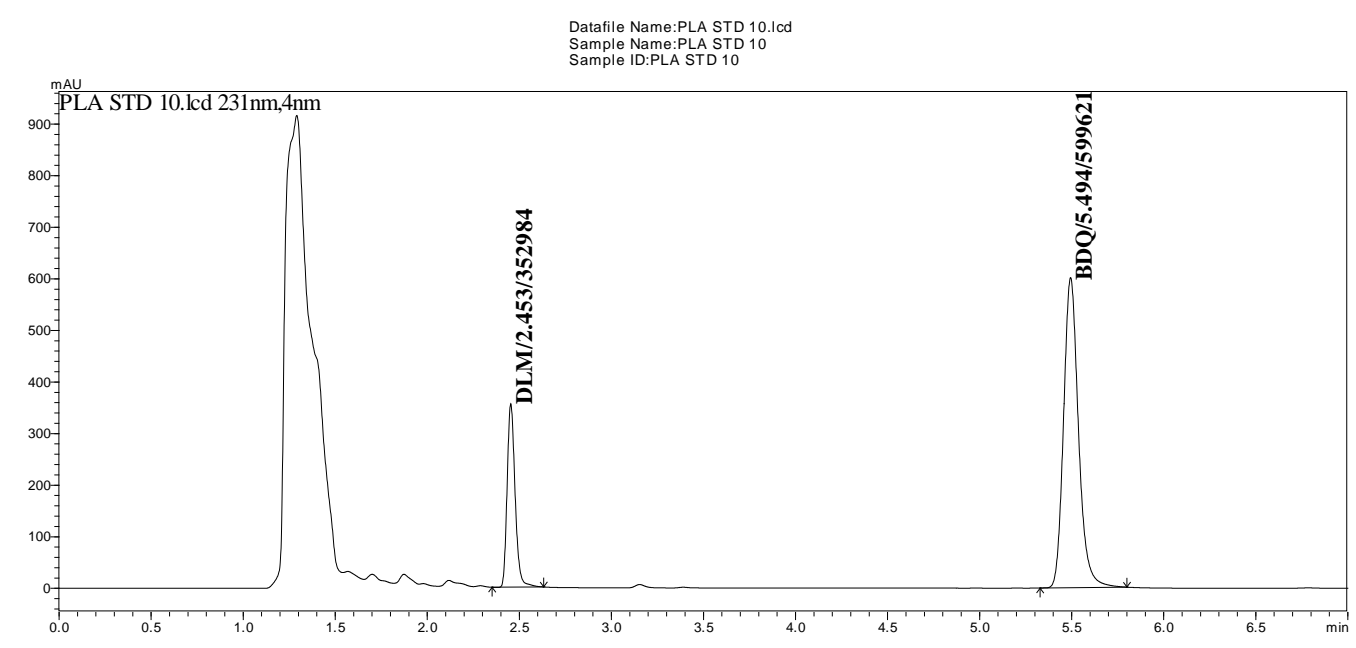

Fig. 1A: Chromatogram of extracted Bedaquiline and Delamanid plasma standard $10.0 \mu \mathrm{g} / \mathrm{ml}$

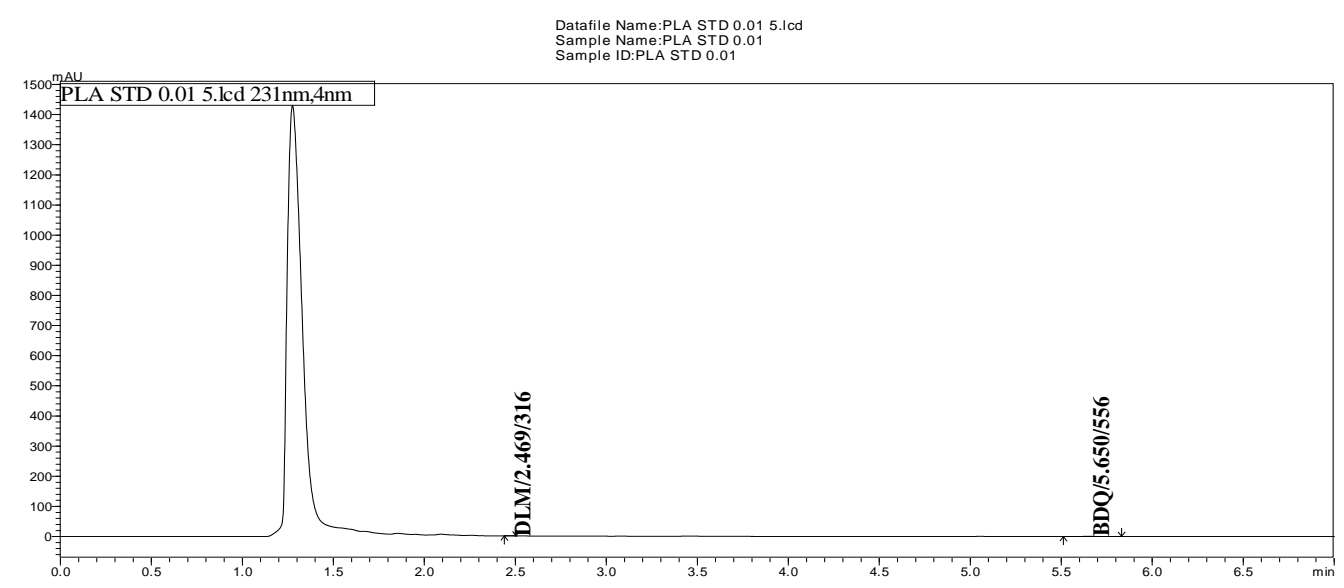

Fig. 1B: Chromatogram of extracted Bedaquiline and Delamanid plasma standard $0.01 \mu \mathrm{g} / \mathrm{ml}$ 


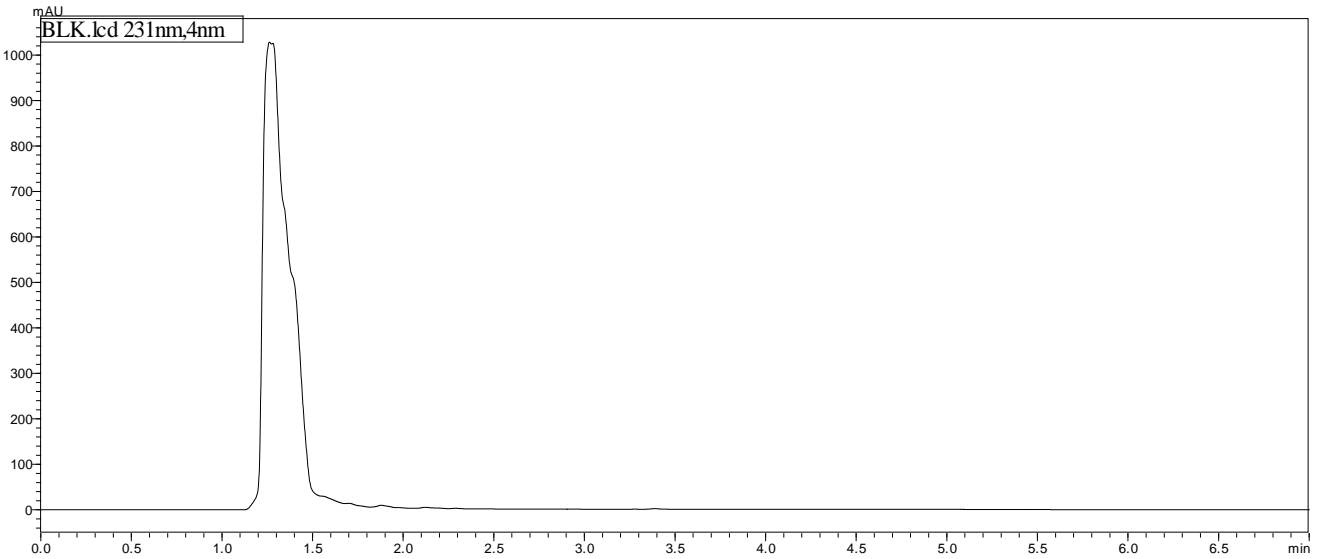

Fig. 1C: Chromatogram of extracted blank plasma

In the present method, BDQ and DLM concentrations ranging from 0.01 to $10 \mu \mathrm{g} / \mathrm{ml}$ were checked for linearity. These concentrations span the range of clinical interest. The calibration curve parameters of BDQ and DLM from six individual experiments for standard concentrations ranging from 0.01 to $10.0 \mu \mathrm{g} / \mathrm{ml}$ showed a linear relationship (fig. $2 \mathrm{~A}$ and $2 \mathrm{~B}$ ). The mean correlation coefficient (R), coefficient of determinants (R2), slope and intercept values were $0.9996,0.9998,61583$ and-486.84 for BDQ and 0.9999, 0.9999, 35943 and-344.63 for DLM respectively.

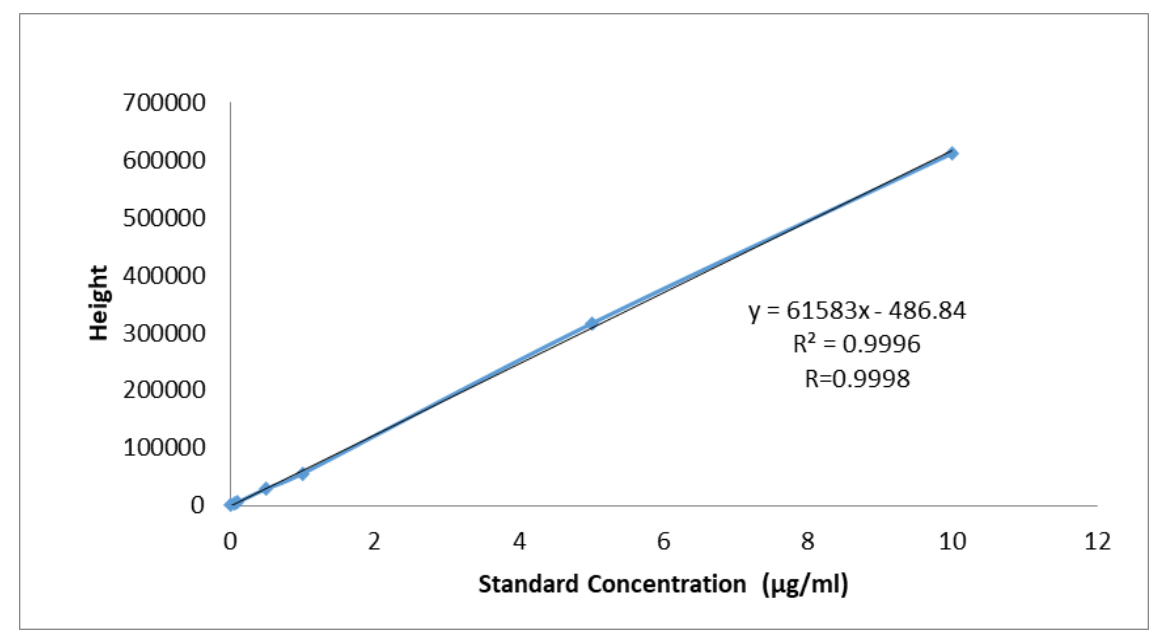

Fig. 2A: Calibration curve of plasma Bedaquiline

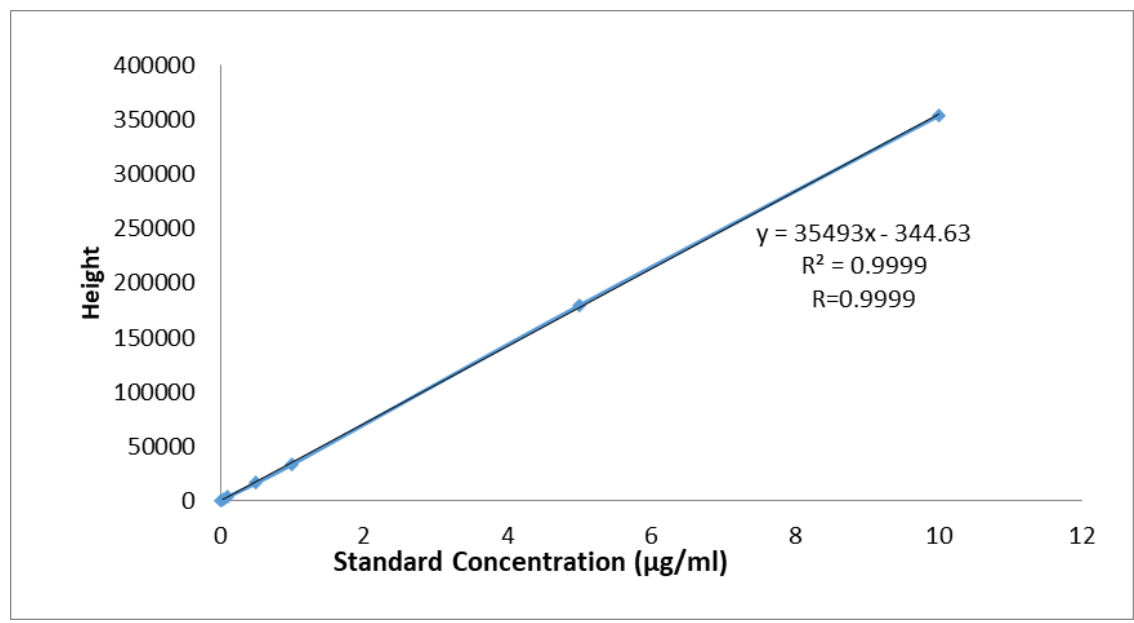

Fig. 2B: Calibration curve of plasma delamanid 
The linearity and reproducibility of the various standards used for constructing calibration graphs for plasma BDQ and DLM are given in (table 1). The intra-and inter-day relative standard deviation (RSD) for standards containing 0.01-10.0 $\mu \mathrm{g} / \mathrm{ml}$ ranged from 2.41 to $4.92 \%$ and 2.59 to $5.35 \%$ for BDQ and 0.76 to $4.41 \%$ and 2.18 to $5.99 \%$ DLM respectively. The accuracy of plasma BDQ and DLM concentrations ranged from 93\% to 102 and are given in (table 2).

Table 1: Linearity and reproducibility of plasma bedaquiline and delamanid standards

\begin{tabular}{|c|c|c|c|c|}
\hline \multirow{3}{*}{$\begin{array}{l}\text { Standard } \\
\text { concentration } \\
(\mu \mathrm{g} / \mathrm{ml})\end{array}$} & \multicolumn{4}{|c|}{ Mean peak height+SD(\%RSD) } \\
\hline & \multicolumn{2}{|c|}{ Delamanid } & \multicolumn{2}{|l|}{ Bedaquiline } \\
\hline & Within day $(n=5)$ & Between day $(n=5)$ & Within day $(n=5)$ & Between day $(n=5)$ \\
\hline 10 & $353636+2685.57(0.76)$ & $365778+15866.66(4.34)$ & $611653+20414(3.34)$ & $536320+13868(2.59)$ \\
\hline 5 & $179418+7311.22(4.07)$ & $171708+3746.49(2.18)$ & $315928+11387(3.6)$ & $264907+10772(4.07)$ \\
\hline 1 & $33518+713.34(2.13)$ & $30421+1582.17(5.2)$ & $55756+1342(2.41)$ & $51281+2742(5.35)$ \\
\hline 0.5 & $16491+340.33(2.06)$ & $15118+462.14(3.06)$ & $29592+822(2.78)$ & $25343+709(2.8)$ \\
\hline 0.1 & $3565+100.89(2.83)$ & $3200+82.12(2.57)$ & $5764+272(4.72)$ & $5009+181(3.62)$ \\
\hline 0.05 & $1856+83.68(4.51)$ & $1689+101.26(5.99)$ & $3242+159(4.92)$ & $2553+75(2.93)$ \\
\hline 0.01 & $413+16.41(3.97)$ & $308+12.67(4.12)$ & $623+20(3.3)$ & $501+23(4.66)$ \\
\hline
\end{tabular}

$S D=$ Standard deviation, $R S D=$ Relative standard deviation, $n=$ number,$\mu g / m l=$ microgram per millilitre

Table 2: Accuracy of plasma bedaquiline and delamanid

\begin{tabular}{|c|c|c|c|c|}
\hline \multirow[t]{2}{*}{ Standard } & \multicolumn{2}{|l|}{ Delamanid } & \multicolumn{2}{|l|}{ Bedaquiline } \\
\hline & Mean $(n=6)$ & $\%$ & Mean $(n=6)$ & $\%$ \\
\hline 10 & 10.02 & 100 & 9.98 & 100 \\
\hline 5 & 5.11 & 102 & 5.11 & 102 \\
\hline 1 & 0.93 & 93 & 0.93 & 93 \\
\hline 0.5 & 0.47 & 94 & 0.49 & 98 \\
\hline 0.1 & 0.1 & 100 & 0.1 & 100 \\
\hline 0.05 & 0.049 & 98 & 0.051 & 102 \\
\hline 0.01 & 0.010 & 100 & 0.010 & 100 \\
\hline
\end{tabular}

$n=$ number, the precision of the method was further evaluated by analysing plasma samples containing different concentrations of BDQ and DLM (table 3). The RSD for these samples ranged from 94 to $100 \%$ for DLM and 97 to $102 \%$ for BDQ respectively.

Table 3: Precision of plasma bedaquiline and delamanid

\begin{tabular}{llll}
\hline $\begin{array}{l}\text { Actual concentration } \\
(\boldsymbol{\mu g} / \mathbf{m l})\end{array}$ & Delamanid & \multicolumn{2}{l}{ Bedaquiline } \\
\cline { 2 - 4 } 10 & Found concentration $(\boldsymbol{\mu g} / \mathbf{m l})$ mean+SD & \% RSD & Found concentration $(\boldsymbol{\mu g} / \mathbf{m l}) \mathbf{m e a n}+\mathbf{S D}$ \\
0.5 & $10.03 \pm 0.18$ & 100 & $10.0 \pm 0.30$ \\
0.05 & $0.47 \pm 0.01$ & 94 & $0.48 \pm 0.03$ \\
0.01 & $0.05 \pm 0.0$ & 96 & $0.051 \pm 0.01$ \\
\hline
\end{tabular}

$S D=$ Standard deviation, $R S D=$ Relative standard deviation, $n=$ number,$\mu \mathrm{g} / \mathrm{ml}=$ microgram per millilitre

The LOD and LOQ were estimated mathematically from the standard curve equation and were 0.005 and $0.01 \mu \mathrm{gg} / \mathrm{ml}$ for both BDQ and DLM respectively. The method reliably eliminated interfering materials from plasma, yielding a recovery for BDQ and DLM that ranged from $101 \%$ to $107 \%$ for DLM and 98 to $107 \%$ for BDQ respectively (table 4). Stability values were defined in terms of changes in concentration and should be $<15 \%$. BDQ and DLM concentrations were stable in plasma at $-20^{\circ} \mathrm{C}$ up to one month.

Table 4: Recovery of plasma bedaquiline and delamanid

\begin{tabular}{|c|c|c|c|c|c|c|c|c|}
\hline \multirow{2}{*}{$\begin{array}{l}\text { Base } \\
(\mu \mathrm{g} / \mathrm{ml})\end{array}$} & \multicolumn{4}{|c|}{ Delamanid } & \multicolumn{4}{|c|}{ Bedaquiline } \\
\hline & $\begin{array}{l}\text { Added } \\
(\mu \mathrm{g} / \mathrm{ml})\end{array}$ & $\begin{array}{l}\text { Actual } \\
(\mu \mathrm{g} / \mathrm{ml})\end{array}$ & $\begin{array}{l}\text { Obtained } \\
(\mu \mathrm{g} / \mathrm{ml})\end{array}$ & Recovery (\%) & $\begin{array}{l}\text { Added } \\
(\mu \mathrm{g} / \mathrm{ml})\end{array}$ & $\begin{array}{l}\text { Actual } \\
(\mu \mathrm{g} / \mathrm{ml})\end{array}$ & $\begin{array}{l}\text { Obtained } \\
(\mu \mathrm{g} / \mathrm{ml})\end{array}$ & Recovery (\%) \\
\hline 5 & 10 & 7.5 & 7.68 & 102 & 10 & 7.5 & 7.76 & 103 \\
\hline 1 & 5 & 3 & 3.11 & 104 & 5 & 3 & 2.95 & 98 \\
\hline 0.5 & 1 & 0.75 & 0.76 & 101 & 1 & 0.75 & 0.76 & 101 \\
\hline 0.05 & 0.1 & 0.075 & 0.08 & 107 & 0.1 & 0.075 & 0.08 & 107 \\
\hline
\end{tabular}

$\mu \mathrm{g} / \mathrm{ml}=$ microgram per millilitre

\section{Clinical application}

The method described was applied for the determination of BDQ and DLM concentrations in plasma samples from 5 patients who were on treatment for XDR-TB receiving $200 \mathrm{mg}$ BDQ once daily and
$100 \mathrm{mg}$ DLM bi-daily under the BEAT study (fig. 3). Serial blood samples were collected at different time points after drug administration. The mean plasma peak concentration of BDQ and DLM were $3.29 \mu \mathrm{g} / \mathrm{ml}$ and $0.34 \mu \mathrm{g} / \mathrm{ml}$ respectively. 


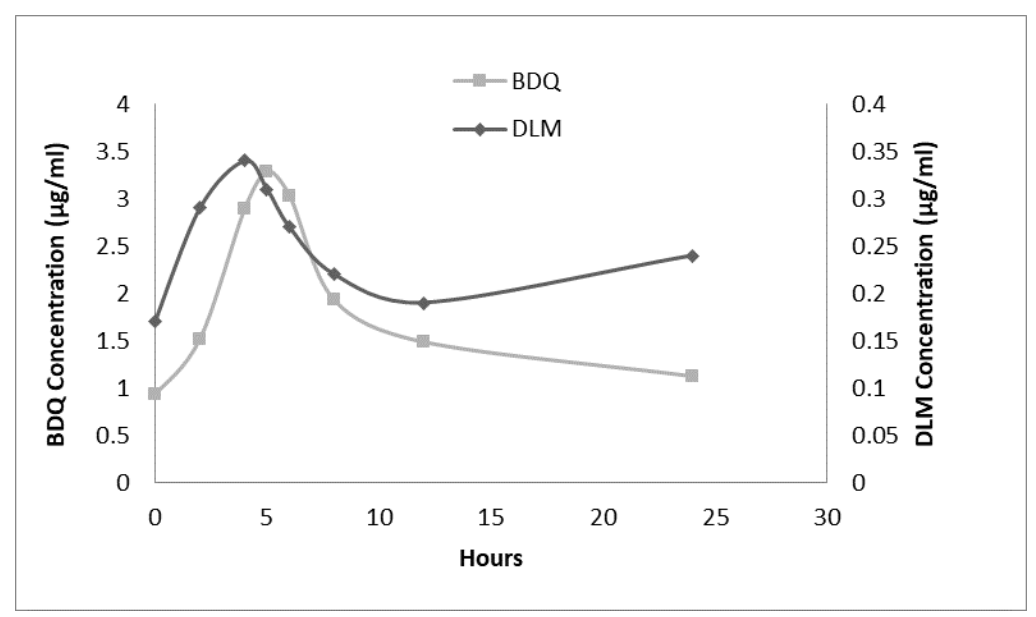

Fig. 3: Plasma bedaquiline and delamanid concentration-time curve

\section{DISCUSSION}

This report describes the design and validation of a simple, specific and sensitive simultaneous analysis method using high-performance liquid chromatography for the determination of BDQ and DLM concentrations in plasma. The method developed showed good accuracy and precision. The calibration curve was linear ranging from 0.01 to $10.0 \mu \mathrm{g} / \mathrm{ml}$ for both BDQ and DLM respectively. The assay spans the concentration range of clinical interest.

Many methods are available that can quantitate plasma BDQ and DLM by LCMS/MS $[2,6,7]$. Meng et al. developed a method to quantitate DLM and its metabolites in plasma using UHPLC-MS/MS [7]. These expensive techniques are out of reach for several laboratories with resource-poor settings. None of the LCMS/MS methods included second-line anti-TB or anti-retroviral drugs in their specificity experiments. Treatment of MDR-TB is always a combination of second-line anti-TB drugs. The available LCMS/MS methods estimate BDQ or DLM separately. The developed HPLC method quantitates both BDQ and DLM simultaneously.

As an evaluation of drug concentrations in relation to the susceptibility of the pathogen may help to explain differences in treatment responses, it is highly desirable that pharmacokinetics should become part of future phase III studies [9]. Therefore estimation of plasma drug concentrations of new drugs such as BDQ and DLM will receive more attention in future pharmacokinetic studies and therapeutic drug monitoring.

\section{CONCLUSION}

In conclusion, we have described a simultaneous method for estimation of BDQ and DLM in plasma, that has the sensitivity to quantitate these drugs from $0.01 \mu \mathrm{g} / \mathrm{ml}$ which is sufficiently enough for future pharmacokinetic studies. This method is reproducible and specific for the determination of BDQ and DLM in human plasma, yielding satisfactory recovery from human plasma. Stability experiments showed that BDQ and DLM were stable up to $30 \mathrm{~d}$ when stored at- $20{ }^{\circ} \mathrm{C}$. This method could be used in clinical studies evaluating drug exposure and correlating with treatment outcome.

\section{ACKNOWLEDGEMENT}

The authors gratefully acknowledge the funding of USAID and STOP TB Partnership through BEAT Study. The authors also thank Janssen Pharmaceuticals (Belgium) for providing BDQ pure powder and Otsuka Pharmaceuticals (Japan) for gifting DLM pure powder. The technical help rendered by Mr. Tamilarasan is acknowledged.

\section{AUTHORS CONTRIBUTIONS}

Dr. A. K Hemanth kumar: Literature search, manuscript preparation and method development, V. Sudha: Method development and validation. A. Vijayakumar: Method development and validation. Dr. C. Padmapriyadarsini: Obtaining BDQ and DLM pure substance, provided fund from BEAT study for consumables and patient samples for method application.

\section{CONFLICT OF INTERESTS}

\section{Declared none}

\section{REFERENCES}

1. Matsumoto $\mathrm{M}$, Hashizume $\mathrm{H}$, Tomishige $\mathrm{T}$, Masanori $\mathrm{K}$, Hidetsugu T, Hirofumi S, et al. OPC-67683, a nitro-dihydroimidazooxazole derivative with promising action against tuberculosis in vitro and in mice. PLoS Med 2006;11:e466.

2. Cuyckens F, Balcaen LIL, De Wolf K, De Samber B, Van Looveren C, Hurkmans R, et al. Use of the bromine isotope ratio in HPLC-ICP-MS and HPLC-ESI-MS analysis of a new drug in development. Anal Bioanal Chem 2008;390:1717-29.

3. Balcaen LIL, De Samber B, De Wolf K, Cuyckens F, Vanhaecke F. Hyphenation of reverse-phase HPLC and ICP-MS for metabolite profiling-application to a novel antituberculosis compound as a case study. Anal Bioanal Chem 2007;389:777-86.

4. Rustomjee R, Diacon AH, Allen J, Venter A, Reddy C, Patientia $\mathrm{RF}$, et al. Early bactericidal activity and pharmacokinetics of the diarylquinoline TMC207 in treatment of pulmonary tuberculosis. Antimicrob Agents Chemother 2008;52:2831-5.

5. Kelly E Dooley, Jeong Gun Park, Susan Swindells, Reena Allen, David W Haas, Yoninah Cramer, et al. Safety, tolerability, and pharmacokinetic interactions of the antituberculous agent TMC207 (bedaquiline) with efavirenz in healthy volunteers: AIDS clinical trials group study A5267. J Acquir Immune Defic Syndr 2012:59:455-62.

6. Jan Willem C Alffenaar, Mathieu Bolhuis, Kai Van Hateren, Marieke Sturkenboom, Onno Akkerman. Determination of bedaquiline in human serum using liquid chromatography-tandem mass spectrometry. Antimicrob Agents Chemother 2015;59:5675-80.

7. Meng M, Smith B, Johnston B, Carter S, Brisson J, Roth SE. Simultaneous quantitation of delamanid (OPC-67683) and its eight metabolites in human plasma using UHPLC-MS/MS. J Chromatogr B 2015;1002:78-91.

8. M Muller, H Stass, M Brunner, JG Muller, E Lackner, HG Eichler. Penetration of moxifloxacin into peripheral compartments in humans. Antimicrob Agents Chemother 1999;43:45.

9. Warner DF, Mizrahi V. Shortening treatment for tuberculosisback to basics. New Engl J Med 2014;371:1642-4. 